\title{
MODIFICATION OF FLUID FLOW EQUATION IN SATURATED POROUS MEDIA
}

\author{
O. O. ALABI, O. I. POPOOLA AND J. A. ADEGOKE
}

(Received 4, March 2008; Revision Accepted 1, June 2009)

\begin{abstract}
Experimental investigations have shown that variation of porosity and hydraulic gradient are responsible for the deviations from Darcy's law, which is perfectly obeyed only when the fluid flow is laminar in porous media. Previous attempts to modify this equation considered only the effects of porosity of surface-active materials such as clay in causing deviations from Darcy's law. In this study, both the effect of porosity of any porous medium and hydraulic gradient from recent experimental data were considered. A general equation for both laminar and nonlaminar or turbulent fluid flow in porous media at any hydraulic gradient is proposed, including the boundary conditions.
\end{abstract}

KEYWORDS: Porous media, laminar flow, non-laminar flow, hydraulic gradient, porosity and permeability.

\section{INTRODUCTION}

In 1901, Forchheimer published a paper in which he noted that Darcy's law is not universally valid for flow, in porous media. Since, then several research papers have been presented on the hydrodynamics of this so called non-Darcy flow.

A look at previous investigations indicate that majority of them found Darcy's law to hold at very low gradients (Meinzer and Fishel, 1934; Fishel, 1935) but with no specification. In 1962, Swartzendruber in reference and re-evaluating of Steams' data (Stearns, 1927) from clay analyses in measurements of flow rate and gradient to assess any effect of clay in causing deviations from Darcy's law and proposed a refined flow equation.' However, clay is not the only surface active immaterial or a porous medium

Considerable progress has been made recently in the analysis of fluid flow through porous media for those situations in which the flow is significantly affected by variations in the porosity and hydraulic gradient. (Popoola et al, 2007) have shown how deviations from Darcy's law is related to porosity and how to determine the maximum gradient for laminar flow vis-a-vis 'Darcy law regime' from porosity, which the fluid is incompressible and the media is saturated.

In the present work certain historical aspects are considered and re-evaluation of recent data given in support of effect of porosity and hydraulic gradient in deviations from Darcy's law. In addition, a refinement of the modified equation will be developed.

\section{Previous Investigations}

King (1898) cited a number of instances in which the flow $q=c_{1} i+c_{2}$ velocity increased more than proportionality with the hydraulic gradient, which implies non-Darcy flow behaviour. Stearns (1927) provided clay analysis in which relationship between flow rate and gradient is given by $q=c_{1} i+c_{2}$ instead of common $q=$ $c i$, where $\mathrm{q}$ is the one-dimensional flow rate of water, is the hydraulic gradient, and $\mathrm{C}_{1}$ and $\mathrm{C}_{2}$ are constants. $\mathrm{He}$ made a conclusion that the presence of appreciable clay appears to be associated with deviations from Darcy's law.

Meinzer and Fishel (1934) and Fishel (1935) found Darcy's law to hold at very low gradients but without any specification.

These are being attempted to modify fluid flow equation in porous media vis-à-vis, Darcy's law. One of them is the modified flow equation proposed by Swartzendruber in 1962. He proposed that

$$
V=M\left[i-I\left(i-e^{-i / 1}\right)\right]
$$

where $\mathrm{V}$ is the macroscopic flow velocity, $\mathrm{i}$ is the hydraulic gradient, $M$ and $I$ are constants. As $i$ increases, equation (1) becomes linear, $\mathrm{V}=\mathrm{Mi}$ and $I$ is simply the $i$ intercept obtained when the linear part of equation (1) is extrapolated back to the $i$ axis.

Hansbo (1960) proposed from consolidation of clay with sand experiment that the velocity - gradient relationship can be written as

$$
V=K i^{n}
$$

for gradient less than limit value $U$ (non-Darcy gradient), $K$ is a constant, and $n$. is on exponent generally greater than 1.

$$
\mathrm{V}=\mathrm{K}\left(i-i_{0}\right)
$$

for gradient $i$ exceeds $i_{1}$ and

$$
V=K i
$$

when $i=i_{1}$

This suggests that due to effect of porosity, the fluid flow equation varies with gradient being considered. However the relationship between porosity and gradient limit ii is not given, thus the gradient limit value could not

O. O, Alabi, Department of Mathematical and Physical Sciences, College of Science, Engineering and Technology, Osun State University, P.M.B. 4494, Osogbo, Osun State Nigeria

O. I. Popoola, Physics Department, University of Ibadan, Oyo State, Nigeria.

J. A. Adegoke, Physics Department, University of Ibadan, Oyo State, Nigeria. 
be specified for a particular medium of porosity $\phi$.

Forchheimer (1901) conducted experiments on a sand-box model and proposed on equation in a quadratic form

$$
I=a V+b V^{2}
$$

The non-Darcy regime of flow, where $a$ and $b$ are the coefficients determined by properties of the fluid and porous media and also known as linear and nonlinear parameter. $\mathrm{J}$ is the hydraulic gradient and $V$ is the velocity. Later on, investigation carried out by Ward (1964) and Ahmed and Sunada (1969) expressed dimensionally the equation for both laminar and turbulent flow in porous medias as

$$
I=\frac{\mu V}{\rho g k}+\frac{C_{w} V^{2}}{g \sqrt{k}}
$$

Where

$$
\begin{aligned}
& \mathrm{C}_{\mathrm{w}}=\text { media constants, }{ }^{\wedge}=\text { viscosity; } \\
& \rho=\text { density of the fluid; } \\
& \mathrm{k}=\text { permeability; and } \\
& \mathrm{g}=\text { acceleration due to gravity. }
\end{aligned}
$$

Comparing Forchheiner equation (5) with equation (6), ward obtained expression for $a$ and $b$ as

$$
a=\frac{\mu}{\rho g k}
$$

and

$$
b=\frac{C_{w}}{g \sqrt{k}}
$$

However, the studies which proposed at equation (5) and (6) considered only porosity as factor that responsible for non-Darcy flow, whereas this is not true. Cedergreen (1976) found that when water is flowing in highly permeable materials, the condition can vary from laminar or nearly laminar flow at very small gradients to semi-turbulent or nearly turbulent as the gradient becomes larger. This implies that both permeability which is a function of porosity of the media and gradient under which the fluid is flowing should be considered in proposing a general equation for fluid in porous media.

\section{Recent Investigations and Results}

It has been found that there is a deviation from 'original' Darcy's law, $q=c i$ for porous media of lower porosity. This deviation from Darcy's law (or gradient intercept) increases with decreasing porosity (Popoola et al, 2007). The relationship between volume flux, $q$ and hydraulic gradient $i$ was found to be

where

$$
q=C_{1} i+C_{2}
$$

$C_{1}$ and $C_{2}$ are empirical constant

$C_{i}=$ hydraulic conductivity and

$\mathrm{C}_{2}=$ intercept, (which is negative)

This result is contrary to Darcy's law which was found to be $q=\mathrm{Ci}$ in respective of gradient at which fluid is flowing and porosity of the media. when $q=0$ in equation

Let $i=i$ (measure of deviation from Darcy's law) will be given as

\begin{tabular}{|c|c|c|c|c|}
\hline Sample & Porosity & Hydraulic conductivity $\left(\mathrm{ms}^{-1}\right)$ & Permeability $\left(m_{2}\right)$ & Meas \\
\hline $\bar{A}$ & $0.250 \pm 0.010$ & $0.46 \times 10^{-4}$ & $0.47 \times 10^{-11}$ & 0.76 \\
\hline B & $0.333 \pm 0.002$ & $1.04 \times 10^{-4}$ & $1,06 \times 10^{-11}$ & 0.26 \\
\hline C & $0.364 \pm 0.001$ & $1.14 \times 10^{-4}$ & $1.16 \times 10^{-11}$ & 0.2 \\
\hline $\mathrm{D}$ & $0.400 \pm 0.001$ & $1.63 \times 10^{-4}$ & $1.67 \times 10^{-11}$ & 0.0 \\
\hline E & $0.420 \pm 0.010$ & $3.16 \times 10^{-4}$ & $3.23 \times 10^{-11}$ & U \\
\hline
\end{tabular}

$i^{\prime}=\mathrm{C}_{2} / \mathrm{C}_{1}$

and it was found experimentally that deviation ? $\mathrm{i}$ is related to porosity, with equation (Popoola et al., 2007) $i^{\prime}=16.5 \phi^{2}+15.2 \phi+3.5$

Table 1: Porosity, hydraulic conductivity, permeability and measure of deviation various samples (Popoola et al., 2007) 


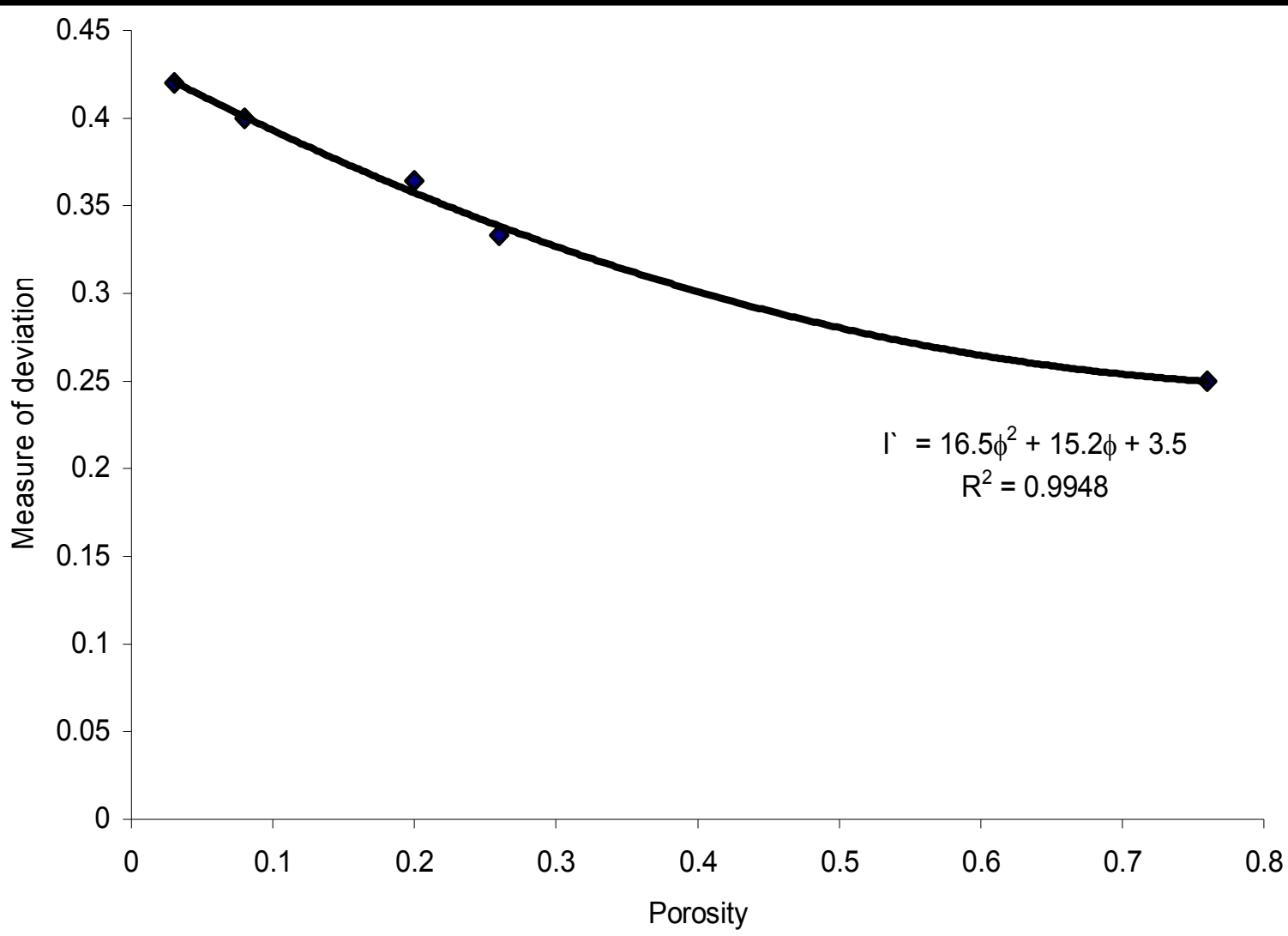

Fig. 1: Graph of Measure of Deviation against Porosity (Popoola et al., 2007)

In another experiment with the same samples, but subjected to both low and higher gradients (1.15 15.00), from which the relative permeabilities were determined. The result shows that their relative permeability increases with increasing porosity but decreases with increasing gradient. It was also found that the vertical fluid flow in any porous medium is laminar or nearly laminar as much as the hydraulic gradient is less than or equal to 1.04 . The maximum hydraulic gradient $i_{m}$ for laminar flow is related to porosity with polynomial equation (Popoola et al., 2007). $i_{m}=6.0008 \phi^{2}-8.6326 \phi+3.679$

Table 2: Maximum gradient for laminar flow for different porosity of Popoola et al (2007)

\begin{tabular}{lcc}
\hline SamplePorosity & Maximum gradient $\left(\boldsymbol{i}_{\mathrm{m}}\right)$ \\
\hline A & $0.250 \pm 0.010$ & 1.88 \\
B & $0.333 \pm 0.002$ & 1.55 \\
C & $0.364 \pm 0.001$ & 1.25 \\
D & $0.400 \pm 0.001$ & 1.15 \\
E & $0.420 \pm 0.010$ & 1.15 \\
\hline
\end{tabular}




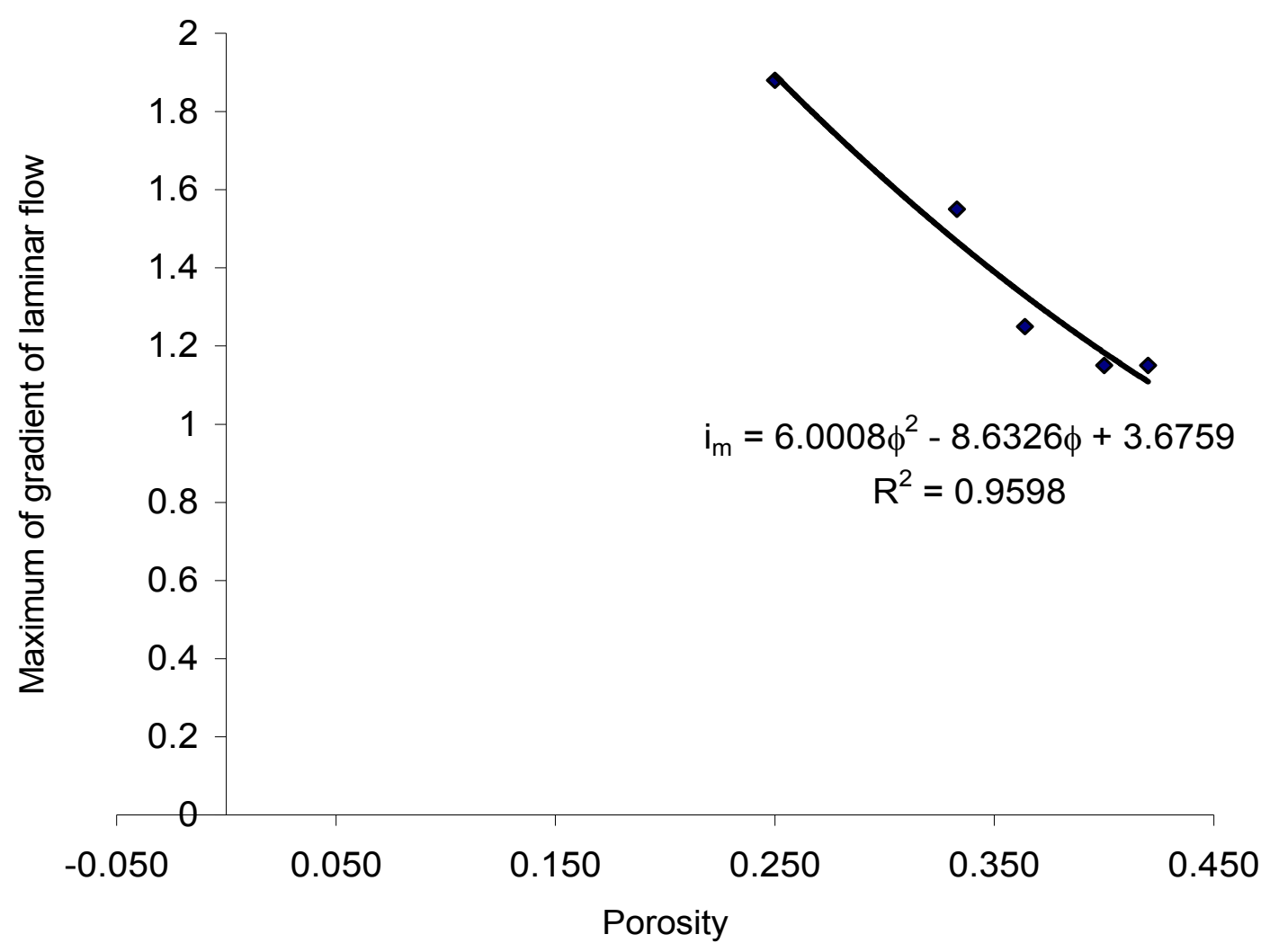

Fig. 2: Graph of Maximu gradient against Porosity (Popoola et al., 2007)

In addition, a general expression (or equation) that take care of both laminar and turbulent flow of fluid in porous media was proposed to be

$\mathrm{q}=\mathrm{k}_{\mathrm{o}}$ ic [Popoola et al., 2007))

where

$q$ = volume flux;

$\mathrm{k}_{\mathrm{o}}=$ 'true' Darcy permeability obtained at very small gradient, $\mathrm{i}_{\mathrm{o}}$ and

$c=\left(i_{o} / 1_{r}\right)=$ correction factor, which varies with relative hydraulic gradient, $i_{r}$ and $\mathrm{n}$ is empirical constant between 0 and 1

As a result of above discussed deviation from Darcy's law which increases with decreasing porosity and non-laminar (or turbulence) flow at higher gradients, there is a need to seek for a general equation that can takes of these limitations or deviations.

\section{DISCUSSION}

The present result shows that the volume flux $q$ and hydraulic gradient $i$ are related with expression, $q=$ $\mathrm{C}_{1} i+\mathrm{C}_{2}$ which is in support of Steam's earlier proposed relationship. This shows that 'simple' relationship, $q=c i$ is not true for all porous media especially the media of lower porosity. Thus, effect of porosity must be considered in formulating a general equation that take care of this deviations. Also, it was observed that permeabilities of a media is not constant in all gradients, but there is decreasing in relative permeability with increasing gradient. This shows that there is a maximum gradient, $\mathrm{i}_{\mathrm{m}}$ at which laminar flow can be achieved for a medium of porosity $\phi$. Gradients beyond this maximum gradient will produce semi-turbulent or turbulent flow. This result is in support of Cedergreen conclusion which stated that fluid flow becomes turbulent as gradient becomes larger. Thus, a modified general fluid flow equation that can take care of these laminar can be stated as

$q=k_{o}\left(\left(\frac{i_{o}}{i_{r}}\right)^{n} i_{r}-i\right)$

$q=$ are dimensional volume flux

= gradient intercept (it is a measure of deviation from Darcy's law)

which increases with decreasing porosity.

$n=$ is an exponent from 0 to 1 , which is a function of porosity.

$C=\left(\frac{i_{o}}{i_{r}}\right)^{n}=$ correction factor 


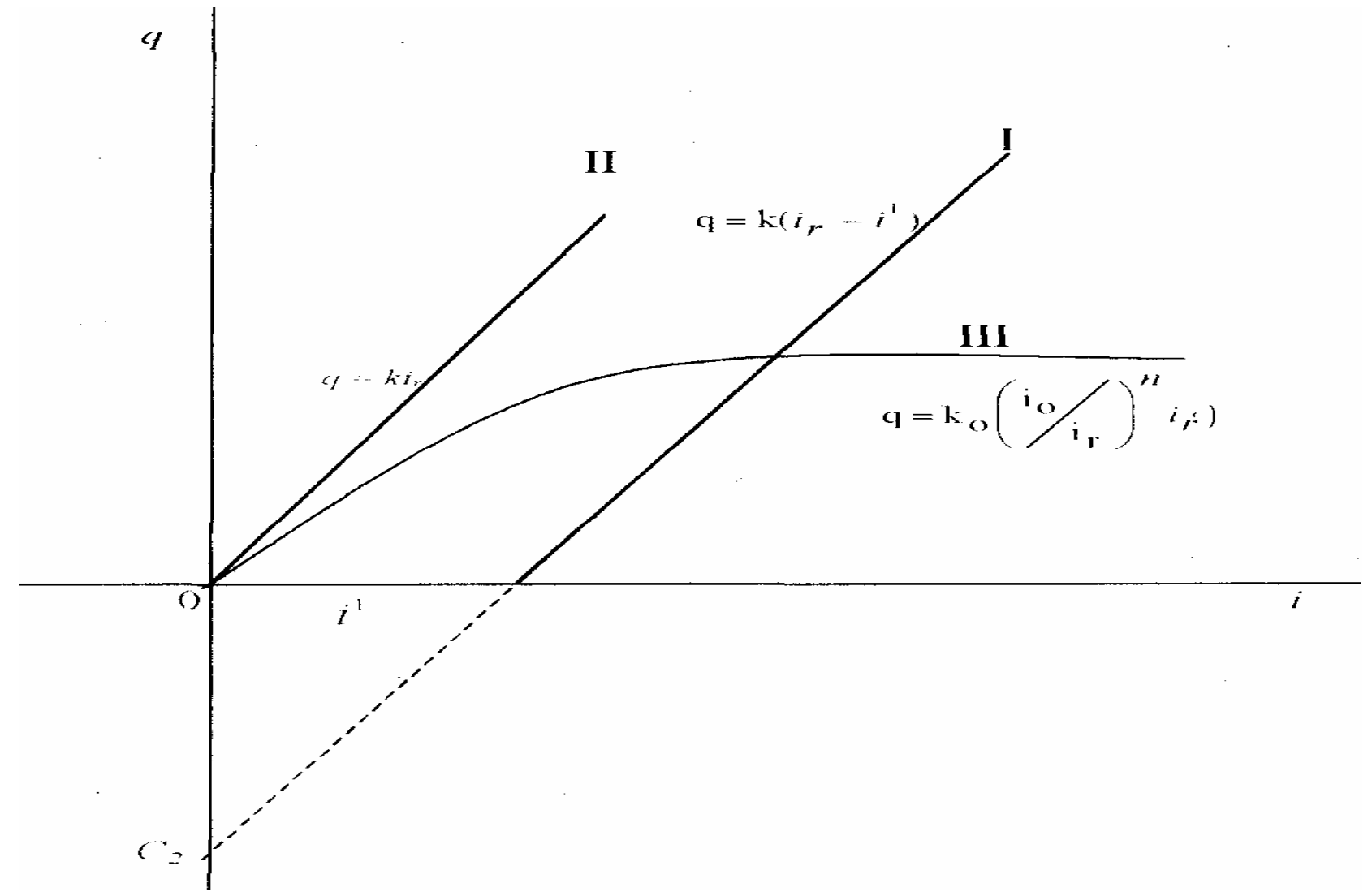

Fig. 3: Illustration of three (3) cases of fluid flow

Curve I: Laminar flow with deviation which increases with decreasing porosity

When

$$
\begin{aligned}
& 0 \leq \mathrm{i}<\mathrm{i}^{\prime} \\
& n=0, i^{\prime}=0 \text { and } \mathrm{k}_{\mathrm{o}}=\mathrm{k} \\
& q=k\left(i_{r}-i^{1}\right)
\end{aligned}
$$

Curve II: Laminar flow without deviation (Darcy's

$$
\begin{aligned}
& \text { law) } \\
& i^{\prime} \leq<\mathrm{i}_{\mathrm{r}}<\mathrm{i}_{\mathrm{m}} \\
& i^{\prime}=0, \mathrm{n}=0, k_{o}=\mathrm{k} \\
& \mathrm{q}=\mathrm{ki} \mathrm{i}_{\mathrm{r}}
\end{aligned}
$$

Curve III: Non-laminar (turbulence) flow with nonconstant permeability.

There is a need for correction factor, $C=\left(i_{o} / i_{r}\right)$ This occur at higher gradients.

$\mathrm{i}<i_{r}>\mathrm{i}_{\mathrm{m}}$

$i=0, n=0$ and $k_{0}=k$

$$
q=k_{o}\left(i_{o} / i_{r}\right)^{n} i_{r}
$$

$C=\left(i_{o} / i_{r}\right)^{n}=$ correction factor decrease from 1 with increasing hydraulic gradient. This should be expected because turbulence increases with increasing hydraulic gradient. Seepage velocity, $\mathrm{V}$ can then be determined from relation $V=q / \phi$ where $q$ is the determined volume ${ }^{\wedge}$ flux from either equation 15,16 or 17 which depends on the condition (or value) of gradient intercept $i$ ' and maximum laminar gradient $\mathrm{i}_{\mathrm{m}}$ in relation to porosity $\phi$ of the medium as stated in equations (11) and (12)

\section{CONCLUSION}

With the suggestion of a modified flow equation, the present authors are not seeking to discredit the monumental contribution which the assertion of Darcy's law has made to understanding of the movement of water in soils and porous media. However, this modification is necessary in order to obtain an equation that is quite accurate enough for all engineering purposes to which it properly apply. Work is currently in progress to evaluate non-Darcy effects in porous media not saturated with water.

\section{REFERENCES}

Ahmed, N and Sunada, D. K., 1967. Non-linear Flow in Porous Media. Journal of Hydr Division, ASCE, 95, ( 6): pp 1847.

Cedergreen, H. R., 1976. Seepage, Drainage and Flow Nets (New York: Willey Interscience Publication).

Fishel, V. C., 1935. Further tests of Permeability with low hydraulic gradients, Trans. Am. Geophys Union, 16, 499 - 503.

Forchheimer, P., 1901. Wasserbewegung Durch Bodeen. Z Ver Deutsch Ing, Vol 45, 1782 - 1788 as in Reddy, N.B.P., 2004. Convergence Effect on the Flow Resistance in Porous Media. NBKR 
Institute of Science and Technology, Vidyanager. P. 36.

Hansbo, S., 1960. Consolidation of clay, with special reference to influence of vertical and drains, Sweden Geotech Inst. Proc 18, p 160.

King, F. H., 1898. Principles and Conditions of the Movement of Groundwater, U. S. Geol. Survey, 91th Ann. Report, Part 2, pp. 59 - 294, as in Swartzendruber, D., 1962. Non-Darcy Flow Behavior in Liquid-Saturated Porous Media. Journal of Geophysical Research, 67; (13): pp. 5206.

Meinzer, 0. E., and Fishel, V. C., 1934. Test of Permeability with low hydraulic gradients, Trans. Am. Geophys Union, 15, 405 -409.

Popoola, O.I., Adegoke, J.A. and Alabi, O.O. 2007. A Laboratory Study of the Effects of Porosity on the Deviation from Darcy's Law in Saturated Porous Media. Research Journal of Applied Sciences 2(8) : 892 - 899.
Popoola, O.I., Adegoke, J.A. and Alabi, O.O. 2007. Modification of Darcy's Law for Turbulent Flow in Saturatec Porous Media. Research Journal of Applied Sciences 2(5) : $642{ }^{\wedge} 645$.

Steams, N., 1927. Laboratory tests on physical properties .water bearing materials, U.S. Geological Survey Water Supply Paper 596, pp.121 - 176.

Swartzendruber, D., 1962. Non-Darcy Flow Behavior in Liquid-Saturated Porous Media. Journal of Geophysical Research 67, (13): pp $67: 5208$.

Ward, J. C., 1964. Turbulent Flow in Porous Media. Journal of Hydr Division, ASCE, 90, (5): p 1. 